\title{
Detecting the Number of Signals in Wireless DS-CDMA Networks
}

\author{
Shahrokh Valaee and Shahram Shahbazpanahi
}

\begin{abstract}
In this paper, a new information theoretic algorithm is proposed for signal enumeration in DS-CDMA networks. The approach is based on the predictive description length (PDL) of the observation vector. The PDL is the length of a predictive code of observations. For signal detection, the PDL criterion is computed for the candidate models and is minimized to determine the number of signals. The proposed technique uses the maximum likelihood (ML) estimate of the correlation matrix. The only information used in the ML estimation of the correlation matrix is the multiplicity of the smallest eigenvalue. The PDL algorithm has a signal-to-noise ratio resolution threshold that is smaller than that of the minimum description length (MDL). The proposed method can be used on-line and can be applied to time-varying and non-stationary systems.
\end{abstract}

Index Terms-DS-CDMA networks, cellular networks, wireless local area networks (WLANs), multiuser detection, signal enumeration, information theoretic techniques, predictive description length.

\section{INTRODUCTION}

D IRECT sequence code division multiple access (DSCDMA) has widely been used in wireless communications. A DS-CDMA signal is formed by multiplying each data bit by the signature waveform allocated to the user. In an ideal case, the signature waveforms form an orthogonal set. Therefore, the data bit is extracted by multiplying the received signal by the corresponding signature waveform. However, in practice, the signature waveforms are rarely orthogonal. For instance, in cellular networks, the received signal is the superposition of the actual signal and inter-cell and intra-cell interference. The interference is generated by the transmitters that use a signature waveform that is not orthogonal to the signature waveform of the desired signal. Nonetheless, if the signature waveforms are selected at transmitters as orthogonal signals, channel impairments such as random delay and multipath transmission may cause the signals to be nonorthogonal at the receiver.

Another example of the application of DS-CDMA systems is in WLANs. In practice, it is possible to operate several WLANs in the same environment by using a unique DSCDMA signal for each WLAN. Since wireless terminals operate on different WLANs, the signal received by a receiver

Paper approved by A. F. Naguib, the Editor for Space-Time and CDMA of the IEEE Communications Society. Manuscript received August 3, 2003; revised May 5, 2006.

S. Valaee is with the Department of Electrical and Computer Engineering, University of Toronto, 10 King's College Road, Toronto, Ontario, Canada, M5S 3G4 (e-mail: valaee@comm.utoronto.ca).

S. Shahbazpanahi is with the Faculty of Engineering and Applied Science, University of Ontario Institute of Technology, 2000 Simcoe Street North, Oshawa, Ontario, Canada, L1H 7K4 (e-mail: shahram.shahbazpanahi@uoit.ca).

Digital Object Identifier 10.1109/TCOMM.2008.030521. is the combination of the signals transmitted by terminals communicating on different WLANs, and since the DS-CDMA signals are generated on different WLANs, they may not be orthogonal, hence, the signal of each WLAN will act as an interference for other WLANs.

In both examples above, the channel is interferencelimited. In such systems, the performance of the conventional receivers - in terms of bit-error-rate (BER) - is very poor. Multiuser detectors [1] can be used to combat the degradation of performance of conventional detectors. A multiuser detector can significantly reduce the BER. Multiuser detectors need to know the true number of signals and their corresponding signature waveforms. If only the signature waveform of the signal-of-interest is known to the receiver, a blind multiuser detector [2] should be used. An example of this case is the reception of downlink signals at a mobile terminal. A blind multiuser detector uses a signal subspace method to detect the transmitted data. The signal subspace methods assume that the true number of transmitted signals is known.

In many applications, the true number of signals is not known and should be estimated prior to multiuser detection. Here, we present two such examples. In wireless cellular networks, the true number of signals is not known at mobile terminals. Therefore, if signal subspace-based multiuser detectors are to be used, the true number of signals should be estimated. A similar problem exists in uplink since the number of interfering signals emanated from wireless terminals connected to neighboring cells is not known at the base station.

In WLANs, since each WLAN is an autonomous system, it is very difficult to determine the number of active users (WLANs) and communicate it to all wireless terminals. Therefore, effective techniques should be developed to detect the number of signals by observing the waveform of the received signal. In this paper, we introduce a novel technique to enumerate DS-CDMA signals that are used in both cellular networks and WLANs.

In an enumeration problem, several hypotheses-each corresponding to a particular number of signals-are examined and the best hypothesis is selected using a certain cost. In this paper, we use an information theoretic approach to detect the number of DS-CDMA signals. Recently, much attention has been given to information theoretic criteria [3]-[11]. A popular information theoretic technique is the minimum description length (MDL) [12]. The MDL is based on minimizing the length of the code required to describe data. Code length minimization is appropriate for model selection since the model, which best fits the data, is the one that gives the most information about it; having more information results in a 


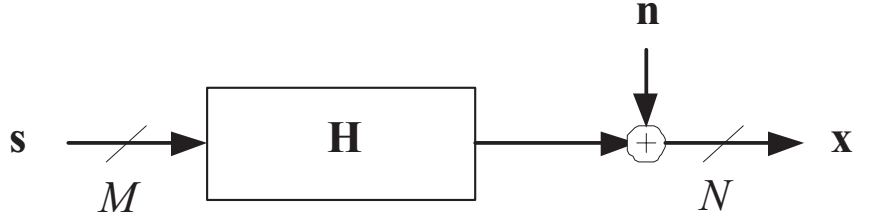

Fig. 1. The block diagram of the system.

smaller code length. Coding of data in the MDL criterion is performed in two steps. First, data is encoded using a uniquely decodable prefix code. Then, the parameter vector is encoded and added as a preamble to the codeword of data. Therefore, the MDL cost consists of two parts that correspond to the loglikelihood function of the observation vector and an additive over-parameterization term. The over-parameterization term in MDL represents the number of digits required to encode the parameter vector to an optimal precision [13]. It has been shown that the MDL is consistent [14]. MDL can only be applied to a batch of data and therefore cannot be used in on-line procedures.

Most MDL approaches in the literature assume that the transmitted signals are Gaussian. This assumption has been used even for the cases where the signals are known to be non-Gaussian. Recently, in [15], the MDL estimator has been modified so that it can be applied to non-Gaussian signals. It has been shown in [15] that the modified MDL approach not only improves the enumeration performance, but also enhances the resolution capacity; that is, the number of signals that can be detected by the modified MDL technique is larger than the length of the received data vector. However, the method of [15] requires that the ML estimates of source signatures be obtained. In general, the ML estimate of such signature waveforms can be computationally very expensive.

In this paper, we develop an algorithm based on the predictive description length (PDL) [13] [16]. The PDL criterion is the cumulative log-likelihood function of the observation vectors such that at each time instant, the maximum likelihood (ML) estimate of the parameter based on the past data is used in the probability distribution function. In the MDL principle, the restriction of coding to a two-step scheme increases the code length. It has been shown that the PDL achieves the shortest code length for data relative to the generating model class [16]. The PDL technique has a structure that is suitable for on-line tracking of time-varying systems.

We apply the PDL principle to a system with the block diagram shown in Fig. 1. In many applications, the observation vector can be represented as the output of a multipleinput/multiple-output channel with an additive white Guassian noise

$$
\mathbf{x}=\mathbf{H s}+\mathbf{n}
$$

where $\mathbf{x}$ is the $N \times 1$ observation vector, $\mathbf{H}$ is the $N \times M$ channel transfer matrix, $\mathbf{s}$ is the $M \times 1$ signal vector and $\mathbf{n}$ is the $N \times 1$ additive noise vector. $M$ is the dimension of the input signal and $N$ is the dimension of the channel output. In (1), $M<N$ and $\mathbf{H}$ is a full-column-rank matrix; that is, the column vectors of $\mathbf{H}$ are linearly independent. This is a valid assumption in many applications.
There are several applications where the model (1) holds. Two examples are the complex envelope representation of array output signals in a multi-antenna array [17], and temporal representation of discrete signals in harmonic resolution [18]. Another interesting example arises in DS-CDMA networks. In this paper, we will develop a signal enumerator scheme for DS-CDMA networks. However, the proposed technique is general and can be applied to all applications that have the observation vector represented as (1).

\section{DS-CDMA SIGNALS}

In an $M$-user synchronous DS-CDMA system, the received continuous-time baseband signal can be represented as

$$
x(t)=\sum_{n=-\infty}^{\infty} \sum_{m=1}^{M} \alpha_{m} b_{m}(n) h_{m}\left(t-n T_{s}\right)+v(t)
$$

where $\alpha_{m}$ is the received signal amplitude of the $m$ th user, $b_{m}(n)$ is the $n$th data symbol of this user, $h_{m}(t)$ is its signature waveform, $T_{s}$ is the symbol period, and $v(t)$ is the zero-mean additive random noise process with the variance $\sigma^{2}$. In this paper, we consider the short spreading code case, i.e., it is assumed that the chip sequence period is the same as the symbol period. Furthermore, we assume that for each user, the data symbols are independent random variables which are equally likely drawn from a finite alphabet. We further assume that the spreading codes of all users have the same length and the system is single-rate.

We model the channel for each user as an FIR filter whose impulse response is much shorter than the symbol period $T_{s}$, so that in each symbol period, only a few chips are affected by inter-symbol-interference (ISI) [19]. This model is frequently used in wireless communications and can be easily achieved by adjusting the symbol rate $T_{s}$. For each user, the number of those chips affected by the ISI is indeed equal to the length of the user channel impulse response. We assume that the maximum length of the user channel impulse response is $L_{c} T_{c}$, where $T_{c}$ is the chip period. Because the duration of the channel impulse response is assumed to be comparable to the chip period $T_{c}$, there is a substantial inter-chip-interference (ICI) [20]. Using these assumptions, the signature waveform of the $m$ th user is given by

$$
h_{m}(t)=\sum_{l=0}^{L-1} c_{m}(l) g_{m}\left(t-l T_{c}\right)
$$

where $\mathbf{c}_{m}=\left[c_{m}(0), c_{m}(1), \ldots, c_{m}(L-1)\right]^{T}$ is the user spreading code vector, $g_{m}(t)$ is its chip waveform convolved with the channel impulse response, $L$ is the spreading factor (the number of chips per symbol), $T_{c}=T_{s} / L$ is the chip period, and the superscript $(\cdot)^{T}$ stands for the transposition. In an ICI-free scenario, $g_{m}(t)$ spans only one chip, while in practice, due to channel dispersion, $g_{m}(t)$ can span several chips and this causes ICI.

Sampling (2) at $t=k T_{s}+p T_{c}$ for $p=0,1,2, \ldots, L-1$ and ignoring the first $L_{c}$ samples, which are contaminated by ISI, we can use vector notation to write the received data vector as

$$
\mathbf{x}_{k}=\sum_{m=1}^{M} \alpha_{m} b_{m}(k) \mathbf{h}_{m}+\mathbf{n}_{k}
$$


where

$$
\begin{aligned}
\mathbf{x}_{k}= & {\left[x\left(k T_{s}+L_{c} T_{c}\right), x\left(k T_{s}+\left(L_{c}+1\right) T_{c}\right), \ldots,\right.} \\
& \left.x\left(k T_{s}+(L-1) T_{c}\right)\right]^{T} \\
\mathbf{h}_{m}= & {\left[h_{k}\left(L_{c} T_{c}\right), h_{k}\left(L_{c} T_{c}+T_{c}\right), \ldots,\right.} \\
& \left.h_{k}\left((L-1) T_{c}\right)\right]^{T} \\
\mathbf{n}_{k}= & {\left[v\left(k T_{s}+L_{c} T_{c}\right), v\left(k T_{s}+\left(L_{c}+1\right) T_{c}\right), \ldots,\right.} \\
& \left.v\left(k T_{s}+(L-1) T_{c}\right)\right]^{T}
\end{aligned}
$$

are the data vector, the signature vector of the $m$ th user, and the noise vector, respectively. In general, the actual user signature is defined as the user spreading code distorted by the channel effect (or, in other words, each signature represents the result of convolution of the corresponding spreading code and the channel impulse response). Now using the following definitions

$$
\begin{aligned}
s_{m}(k) & \triangleq \alpha_{m} b_{m}(k) \\
\mathbf{H} & \triangleq\left[\begin{array}{llll}
\mathbf{h}_{1} & \mathbf{h}_{2} & \cdots & \mathbf{h}_{M}
\end{array}\right]
\end{aligned}
$$

we get

$$
\mathbf{x}_{k}=\mathbf{H s}_{k}+\mathbf{n}_{k}
$$

where $\mathbf{s}_{k}=\left[s_{1}(k), \ldots, s_{M}(k)\right]^{T}$ is the $M \times 1$ signal vector, and $\mathbf{H}=\left[\mathbf{h}_{1}, \ldots, \mathbf{h}_{M}\right]$ is the $N \times M$ matrix of signature waveforms.

It has already been shown in the literature that a low rank data model can be obtained for frequency selective channels, see [19], [21], [22], and [20]. In fact, as was shown in these references, by ignoring the samples affected by intersymbol-interference (which in turn is the result of frequency selectiveness of the channel), one can come up with the low rank data model (10).

The date model in (10) is applicable to both downlink and uplink communications. In both cases, the number of signals should be known, as the subsequent signature estimation techniques (such as signal subspace based methods) require this piece of information. In uplink, the receiver at the basestation should know the number of signals, which includes the signals from the users in its cell as well as the signals from users in the neighboring cells. The number of out-ofcell signals is not known to the base station and should be estimated. In downlink, the received signal by each user also consists of the signals transmitted by the base station to the other users. The number of such users is not known at the receiver and should be estimated. In this paper, we propose an algorithm to find the column rank of $\mathbf{H}$, which is the same as the number of transmitted signals.

Throughout this paper, we assume that the columns of the signature waveform matrix $\mathbf{H}$ are linearly independent. We further assume that the signal snapshots form an independent identically distributed (i.i.d.) sequence of Gaussian random vectors with an unknown covariance matrix $\mathbf{S}^{M}$. The noise samples are assumed to be independent from the signal samples and form an i.i.d. sequence of Gaussian random vectors with an unknown covariance matrix $\sigma^{2} \mathbf{I}_{N}$, where $\mathbf{I}_{N}$ is the $N \times N$ identity matrix. With these assumptions, the observation vector will be a sample of the Gaussian process with zero mean and the correlation matrix

$$
\begin{aligned}
\mathbf{R}^{M} & =E\left[\mathbf{x}_{k} \mathbf{x}_{k}^{T} \mid \mathbf{H}, \sigma^{2}, \mathbf{S}^{M}\right] \\
& =\mathbf{H S}^{M} \mathbf{H}^{T}+\sigma^{2} \mathbf{I}_{N} .
\end{aligned}
$$

Therefore, the probability density function of the observation vector is

$$
f\left(\mathbf{x} \mid \mathbf{R}^{M}\right)=\frac{1}{(2 \pi)^{\frac{N}{2}}\left|\mathbf{R}^{M}\right|^{\frac{1}{2}}} \exp \left(-\frac{1}{2} \mathbf{x}^{T}\left[\mathbf{R}^{M}\right]^{-1} \mathbf{x}\right)
$$

where |.| represents the determinant of the matrix.

It is possible to show that the observation vector can be decomposed into two orthogonal vectors in the signal and noise subspaces [23]. The signal subspace is the subspace spanned by the column vectors of $\mathbf{H}$. If the signal correlation matrix $\mathbf{S}^{M}$ is full-rank, the signal subspace will coincide with the span of the eigenvectors of $\mathbf{R}^{M}$ corresponding to $M$ largest eigenvalues. Note that the dimension of the signal subspace is $M$. The noise subspace is the orthogonal complement of the signal subspace. The dimension of the noise subspace is $N-M$. The objective of this paper is to estimate the dimension of the signal subspace, $M$, given that $M \in \mathcal{N} \triangleq\{0,1, \ldots, N-1\}$.

A direct implication of the signal and noise subspace decomposition technique is that for a high signal-to-noise ratio (SNR), the eigenvalues of the sample correlation matrix, corresponding to signal components, are significantly larger than the noise eigenvalues. Furthermore, the noise eigenvalues of the true correlation matrix are identical and are equal to $\sigma^{2}$. These observations can be used to devise a simple signal enumeration technique by comparing the difference between consecutive eigenvalues. In the sequel, we will refer to this numerator as EIG. We will show that the performance of this enumerator is inferior to that of MDL and PDL. An inherent problem of the EIG enumerator is that it cannot detect the true number of signals when $M=0$.

\section{INFORMATION THEORETIC CRITERIA}

For any $m \in \mathcal{N}$, we construct an appropriate model of order $m$. Assume that each model $m$ is represented by a conditional probability density function $f\left(\mathbf{x} \mid \phi^{m}\right)$ where $\mathbf{x}$ is the observation vector and $\phi^{m}$ is the corresponding parameter vector. The MDL cost for a model of order $m$ over a window of size $K$ is represented by [12]

$$
\operatorname{MDL}_{m}(K)=-\log f\left(\mathbf{X}^{K} \mid \hat{\phi}_{K}^{m}\right)+\frac{d_{m}}{2} \log K
$$

where $\mathrm{X}^{K}$ is the $N \times K$ matrix of observations up to time $K, \hat{\phi}_{K}^{m}$ is the ML estimate of the parameter vector $\phi^{m}$ using $K$ observation vectors, $d_{m}$ is the number of free elements for the model of order $m$, and $f(. \mid$.) is the generating model class (conditional probability density function). In (14), the first term is the log-likelihood function of the observation vectors and the second term is the over-parameterization factor-a two step encoding scheme.

The PDL cost of the observation vectors $\mathbf{x}_{k}, k=1, \ldots, K$, for a model of order $m$ is defined as [16]

$$
\operatorname{PDL}_{m}(K)=-\sum_{k=1}^{K} \log f\left(\mathbf{x}_{k} \mid \hat{\phi}_{k-1}^{m}\right)
$$


where $\hat{\phi}_{k-1}^{m}$ is the ML estimate of the parameter vector using the observations up to time $(k-1)$. The PDL principle is based on the predictive encoding of data. At each time instant, the parameter vector is estimated using the past observations. Therefore, the $k$ th term, $-\log f\left(\mathrm{x}_{k} \mid \hat{\phi}_{k-1}^{m}\right)$, is indeed the code length of the prediction error [16]. In this paper, the PDL cost is calculated for each model and the smallest is selected as the best-fit model, that is

$$
\hat{M}_{K}=\arg \min _{m} \operatorname{PDL}_{m}(K)
$$

where we have implicitly assumed that the number of signals can be time varying.

The initial point in the recursion (15) is usually chosen arbitrarily. In practice, $f\left(\mathbf{x}_{1} \mid \hat{\phi}_{0}^{m}\right)$ is obtained by selecting $\hat{\phi}_{0}^{m}$ from the uniform distribution $\frac{1}{\phi_{2}-\phi_{1}}$ in $\left[\phi_{1}, \phi_{2}\right]$ for some $\phi_{1}$ and $\phi_{2}$ [24]. In this paper, we take an alternative approach. We collect $N$ snapshots and form the sample correlation matrix using these snapshots. This sample correlation matrix is then used to estimate the parameter vector. The PDL cost is accumulated for all $i=N+1, N+2, \ldots, K$ to find the total code length. Therefore, our formulation of the PDL criterion is

$$
\mathrm{PDL}_{m}(K)=-\sum_{k=N+1}^{K} \log f\left(\mathbf{x}_{k} \mid \hat{\phi}_{k-1}^{m}\right) .
$$

In the following section, we derive the PDL cost to detect the number of linearly independent columns of $\mathbf{H}$ in (1).

\section{Predictive Description Length}

For the model of order $m$, let the channel output signal at the $k$ th time instant be expressed as

$$
\mathbf{x}_{k}=\mathbf{H}_{m} \mathbf{s}_{k}^{m}+\mathbf{n}_{k}^{m}
$$

where $\mathbf{H}_{m}$ is the $N \times m$ matrix of signature waveforms, and $\mathbf{s}_{k}^{m}(t)$ is an $m \times 1$ signal vector, and $\mathbf{n}_{k}^{m}$ is the $N \times 1$ noise vector of model $m$. Assuming that the signal vector $\mathbf{s}_{k}^{m}$ and the noise vector $\mathbf{n}_{k}^{m}$ are independent, the correlation matrix of the observation vector $\mathbf{x}_{k}$ is

$$
\mathbf{R}^{m}=\mathbf{H}_{m} \mathbf{S}^{m} \mathbf{H}_{m}^{T}+\sigma_{m}^{2} \mathbf{I}_{N}
$$

where $\mathbf{S}^{m}$ is the signal autocorrelation matrix and $\sigma_{m}^{2} \mathbf{I}_{N}$ is the noise autocorrelation matrix. Here, we assume that the noise is white with the unknown variance $\sigma_{m}^{2}$ that depends on the selected model.

The conditional probability density function of the observation vector for model $m$ is given by

$$
f\left(\mathbf{x} \mid \mathbf{R}^{m}\right)=\frac{1}{(2 \pi)^{\frac{N}{2}}\left|\mathbf{R}^{m}\right|^{\frac{1}{2}}} \exp \left(-\frac{1}{2} \mathbf{x}^{T}\left[\mathbf{R}^{m}\right]^{-1} \mathbf{x}\right) .
$$

From (20), the PDL for a model of order $m$ at time instant $K \geq N+1$ is given by

$$
\begin{aligned}
\operatorname{PDL}_{m}(K) & =-\sum_{k=N+1}^{K} \log f\left(\mathbf{x}_{k} \mid \hat{\mathbf{R}}_{k-1}^{m}\right) \\
& =\sum_{k=N+1}^{K}\left(\log \left|\hat{\mathbf{R}}_{k-1}^{m}\right|+\mathbf{x}_{k}^{T}\left[\hat{\mathbf{R}}_{k-1}^{m}\right]^{-1} \mathbf{x}_{k}\right)(21)
\end{aligned}
$$

where $\hat{\mathbf{R}}_{k-1}^{m}$ is the ML estimate of the correlation matrix for the model of order $m$ using the observations up to time $(k-$ $1)$; in (21), the constant terms that are independent of the selected model have been removed and the cost function has been multiplied by 2 . The PDL cost is computed for each model and the minimum is chosen to estimate $M$. Indeed, at each time instant $K \geq N+1$, the best model is selected from (16).

In the sequel, the sample correlation matrix is used to obtain the ML estimate of the true correlation matrix. The sample correlation matrix is defined with the recursion

$$
\overline{\mathbf{R}}_{k}=(1-\epsilon) \overline{\mathbf{R}}_{k-1}+\epsilon \mathbf{x}_{i} \mathbf{x}_{i}^{T}
$$

where $0<\epsilon<1$ is usually very small. This definition of the sample correlation matrix is very useful for nonstationary environments. In (22), by varying $\epsilon$, we can have different weights for $\overline{\mathbf{R}}_{k-1}$ and $\mathbf{x}_{k} \mathbf{x}_{k}^{T}$. For large values of $\epsilon$, the sample correlation matrix has a short memory and is sensitive to recent changes of the underlying statistics, and for small values of $\epsilon$, it has a longer memory and sudden changes in the statistics of $\mathbf{x}_{k} \mathbf{x}_{k}^{T}$ cannot be easily detected.

Let $\bar{\lambda}_{k, j}, j=1, \ldots, N$, be the eigenvalues of $\overline{\mathbf{R}}_{k}$ arranged in non-increasing order, and $\overline{\mathbf{v}}_{k, j}, j=1, \ldots, N$ be the corresponding eigenvectors. It is possible to show that the eigenvalues and the eigenvectors of the ML estimator $\hat{\mathbf{R}}_{k}^{m}$ are given by [25]

$$
\begin{aligned}
& \hat{\lambda}_{k, j}= \begin{cases}\bar{\lambda}_{k, j} & \text { for } j=1, \ldots, m, \\
\frac{1}{N-m} \sum_{\ell=m+1}^{N} \bar{\lambda}_{k, \ell} & \text { for } j=m+1, \ldots, N,\end{cases} \\
& \hat{\mathbf{v}}_{k, j}=\overline{\mathbf{v}}_{k, j}(23)
\end{aligned}
$$

In other words, (23) and (24) imply that once the eigenvalues and the eigenvectors of the sample covariance matrix $\overline{\mathbf{R}}_{k}$ are known, the ML estimate of the covariance matrix, $\hat{\mathbf{R}}_{k}^{m}$ can be obtained as a matrix whose eigenvectors are the same as those of $\overline{\mathbf{R}}_{k}$, its largest $m$ eigenvalues are the same as the $m$ largest eigenvalues of $\overline{\mathbf{R}}_{k}$, and its smallest eigenvalue is equal to the arithmetic mean of the smallest $N-m$ eigenvalues of $\overline{\mathbf{R}}_{k}$. Therefore, eigenvalues and eigenvectors of $\overline{\mathbf{R}}_{k}$ can be used to obtain the ML estimate of the correlation matrix, $\hat{\mathbf{R}}_{k}^{m}$ as

$$
\hat{\mathbf{R}}_{k}^{m}=\hat{\mathbf{V}}_{k} \hat{\mathbf{\Lambda}}_{k} \hat{\mathbf{V}}_{k}^{T}
$$

where

$$
\begin{aligned}
& \hat{\mathbf{V}}_{k} \triangleq\left[\overline{\mathbf{v}}_{k, 1} \ldots \overline{\mathbf{v}}_{k, N}\right] \\
& \hat{\mathbf{\Lambda}}_{k} \triangleq\left[\begin{array}{cc}
\operatorname{diag}\left(\bar{\lambda}_{k, 1}, \ldots, \bar{\lambda}_{k, m}\right) & \mathbf{0}_{m, N-m} \\
\mathbf{0}_{N-m, m} & \hat{\sigma}_{m, k}^{2} \mathbf{I}_{N-m}
\end{array}\right]
\end{aligned}
$$

where $\operatorname{diag}\left(\bar{\lambda}_{k, 1}, \ldots, \bar{\lambda}_{k, m}\right)$ is a diagonal matrix with the diagonal entities given in the brackets,

$$
\hat{\sigma}_{m, k}^{2}=\frac{1}{N-m} \sum_{\ell=m+1}^{N} \bar{\lambda}_{k, \ell}
$$

is the ML estimate of the noise variance $\sigma_{m}^{2}$, and $\mathbf{0}_{i, j}$ is an $i \times j$ all-zero matrix.

Using (23) and (24), we formulate the PDL criterion for the model of order $m$ as 


$$
\begin{aligned}
\operatorname{PDL}_{m}(K) & =\sum_{k=N+1}^{K}\left(\sum_{j=1}^{m} \log \bar{\lambda}_{k-1, j}+(N-m) \log \hat{\sigma}_{m, k-1}^{2}\right. \\
& \left.+\mathbf{x}_{k}^{T}\left[\hat{\mathbf{R}}_{k-1}^{m}\right]^{-1} \mathbf{x}_{k}\right) .
\end{aligned}
$$

The PDL criterion is computed for all $0 \leq m \leq N-1$ and the minimum is used to estimate the number of signals.

The PDL cost can also be represented in an alternative way. We subtract $\sum_{k=N+1}^{K} \sum_{j=1}^{N} \log \bar{\lambda}_{k-1, j}$ from all models. Since this term is independent of the selected model, it will not affect the enumeration results. Then, the PDL cost function for model $m$ will be

$$
\begin{aligned}
\operatorname{PDL}_{m}(K) & =\sum_{k=N+1}^{K}\left(\log \left(\frac{\left(\hat{\sigma}_{m, k-1}^{2}\right)^{N-m}}{\prod_{j=m+1}^{N} \bar{\lambda}_{k-1, j}}\right)\right. \\
& \left.+\mathbf{x}_{k}^{T}\left[\hat{\mathbf{R}}_{k-1}^{m}\right]^{-1} \mathbf{x}_{k}\right) .
\end{aligned}
$$

Note that the first term in (29) is the logarithm of the ratio of arithmetic average to the geometric average of the $(N-$ $m$ ) smallest eigenvalues of the sample correlation matrix. The smallest value for this term will be obtained if all $\bar{\lambda}_{k-1, j}, j=$ $m+1, \ldots, N$ are identical. This case corresponds to having a single eigenvalue with the multiplicity $(N-m)$-as expected in a system with $m$ signals. The minimum value of the first term is always obtained for $m=N-1$, and the second term handles over-parameterization.

The MDL cost can also be obtained with a similar approach [14]. The MDL for our problem is

$\operatorname{MDL}_{m}(K)=K \log \left(\frac{\left(\hat{\sigma}_{m, K}^{2}\right)^{N-m}}{\prod_{j=m+1}^{N} \bar{\lambda}_{K, j}}\right)+\frac{m}{2}(2 N-m) \log K$

where the first term measures the multiplicity of the smallest eigenvalue and the second term is the over-parameterization factor.

The method proposed here for the ML estimation of $\mathbf{R}^{m}$ uses the fact that the correlation matrix of the $m$ th model can be expressed as $\mathbf{R}^{m}=\mathbf{Q}^{m}+\sigma_{m}^{2} \mathbf{I}$, where $\mathbf{Q}^{m}$ is a positive semi-definite matrix with rank $m<N$. The structure of $\mathbf{Q}^{m}$ is not used in this approach; the only information utilized is the multiplicity of the smallest eigenvalue of $\mathbf{R}^{m}$. It is possible to show that this technique cannot properly estimate the number of signals when $\mathbf{Q}^{m}$ is rank-deficient (signals are fully correlated). This case is not discussed in the present paper since we assume that the DS-CDMA signals are generated by independent users.

\section{First Order Perturbation}

The PDL criterion, as formulated in (29), needs eigenvalue decomposition and inversion of the correlation matrix that both are computationally expensive. In this section, we provide techniques that can reduce the computational complexity of the PDL algorithm. We use the first order perturbation technique to devise a recursive structure for PDL.

Using the cumulative structure of the PDL algorithm, we have

$$
\operatorname{PDL}_{m}(k)=\operatorname{PDL}_{m}(k-1)+\ell_{m, k}
$$

where $\ell_{m, k}$ is the description length of model $m$ at time instant $k$ and is given by

$\ell_{m, k}=\sum_{j=1}^{m} \log \bar{\lambda}_{k-1, j}+(N-m) \log \hat{\sigma}_{m, k-1}^{2}+\mathbf{x}_{k}^{T}\left[\hat{\mathbf{R}}_{k-1}^{m}\right]^{-1} \mathbf{x}_{k}$.

The estimate of $M$ is then obtained from

$$
\hat{M}_{k}=\arg \min _{m}\left\{\operatorname{PDL}_{m}(k-1)+\ell_{m, k}\right\} .
$$

The recursive structure of (34) allows us to design a trellis representation for the PDL algorithm which can be very useful in time-varying cases [26].

Note that $\left[\hat{\mathbf{R}}_{k-1}^{m}\right]^{-1}$ can be represented by

$$
\left[\hat{\mathbf{R}}_{k-1}^{m}\right]^{-1}=\sum_{j=1}^{N} \frac{1}{\hat{\lambda}_{k-1, j}} \hat{\mathbf{v}}_{k-1, j} \hat{\mathbf{v}}_{k-1, j}^{T} .
$$

Then, using (23) and (24), $\ell_{m, k}$ can be written as

$$
\begin{aligned}
\ell_{m, k} & =\sum_{j=1}^{m} \log \bar{\lambda}_{k-1, j}+(N-m) \log \hat{\sigma}_{m, k-1}^{2} \\
& +\sum_{j=1}^{m} \frac{\left|y_{j, k}\right|^{2}}{\bar{\lambda}_{k-1, j}}+\frac{1}{\hat{\sigma}_{m, k-1}^{2}} \sum_{j=m+1}^{N}\left|y_{j, k}\right|^{2}
\end{aligned}
$$

where $y_{j, k}$ is the projection of the observation vector $\mathbf{x}_{k}$ onto $\overline{\mathbf{v}}_{k-1, j}$, the $j$ th eigenvector of the sample correlation matrix $\overline{\mathbf{R}}_{k-1}$, and is given by

$$
y_{j, k} \triangleq \overline{\mathbf{v}}_{k-1, j}^{T} \mathbf{x}_{k} .
$$

In (36), the eigenvalue decomposition of the sample correlation matrix is needed. A direct calculation of the eigenvalues and eigenvectors is computationally expensive. Here, we use the first order perturbation technique to obtain an estimate of the eigenvalues and eigenvectors [27]. First order perturbation provides a recursive procedure to estimate the eigenvalues and the eigenvectors of the sample correlation matrix $\overline{\mathbf{R}}_{k}$ in terms of the eigenvalues and the eigenvectors of $\overline{\mathbf{R}}_{k-1}$.

In the first order perturbation, we assume that the eigenvalues and the eigenvectors of the sample correlation matrix at a given time $k$ can be found from the corresponding eigenvalues and eigenvectors at time $k-1$ with the following formulas

$$
\begin{aligned}
& \bar{\lambda}_{k, j}=\bar{\lambda}_{k-1, j}+\gamma_{k, j} \epsilon, \\
& \overline{\mathbf{v}}_{k, j}=\overline{\mathbf{v}}_{k-1, j}+\mathbf{u}_{k, j} \epsilon,
\end{aligned}
$$

where $\gamma_{k, j}$ and $\mathbf{u}_{k, j}$ are the perturbation values for the eignevalues and the eigenvectors, respectively. Using (38) and (39) in the eigenvalue decomposition of the sample correlation matrix gives

$$
\begin{array}{r}
{\left[\overline{\mathbf{R}}_{k-1}+\epsilon\left(\mathbf{x}_{k} \mathbf{x}_{k}^{T}-\overline{\mathbf{R}}_{k-1}\right)\right]\left(\overline{\mathbf{v}}_{k-1, j}+\mathbf{u}_{k, j} \epsilon\right)=} \\
\left(\bar{\lambda}_{k-1, j}+\gamma_{k, j} \epsilon\right)\left(\overline{\mathbf{v}}_{k-1, j}+\mathbf{u}_{k, j} \epsilon\right) .
\end{array}
$$

Equating the terms of order $\epsilon$ at the left-hand-side and the right-hand-side of (40) results in

$\left(\mathbf{x}_{k} \mathbf{x}_{k}^{T}-\overline{\mathbf{R}}_{k-1}\right) \overline{\mathbf{v}}_{k-1, j}+\overline{\mathbf{R}}_{k-1} \mathbf{u}_{k, j}=\bar{\lambda}_{k-1, j} \mathbf{u}_{k, j}+\gamma_{k, j} \overline{\mathbf{v}}_{k-1, j}$. 
Multiplying both sides of (41) from left with $\overline{\mathbf{v}}_{k-1, j}$ and using the orthonormality of the set $\left\{\overline{\mathbf{v}}_{k-1, j}\right\}$ gives

$$
\overline{\mathbf{v}}_{k-1, j}^{T}\left(\mathbf{x}_{k} \mathbf{x}_{k}^{T}-\overline{\mathbf{R}}_{k-1}\right) \overline{\mathbf{v}}_{k-1, j}=\gamma_{k, j} .
$$

Using the eigen-decomposition of the sample correlation matrix and the orthonormality of the eigenvectors gives

$$
\left|y_{j, k}\right|^{2}=\overline{\mathbf{v}}_{k-1, j}^{T} \mathbf{x}_{k} \mathbf{x}_{k}^{T} \overline{\mathbf{v}}_{k-1, j}=\bar{\lambda}_{k-1, j}+\gamma_{k, j}
$$

Therefore, the PDL cost can be represented by

$$
\begin{aligned}
\ell_{m, k} & =\sum_{j=1}^{m} \log \bar{\lambda}_{k-1, j}+(N-m) \log \hat{\sigma}_{k-1}^{2} \\
& +\sum_{j=1}^{m} \frac{\gamma_{k, j}}{\bar{\lambda}_{k-1, j}}+\frac{1}{\hat{\sigma}_{k-1}^{2}} \sum_{j=m+1}^{N} \gamma_{k, j}
\end{aligned}
$$

where the constant terms (independent from the model order $m$ ) have been removed, and $\gamma_{k, j}$ is found from

$$
\gamma_{k, j}=\overline{\mathbf{v}}_{k-1, j}^{T} \mathbf{x}_{k} \mathbf{x}_{k}^{T} \overline{\mathbf{v}}_{k-1, j}-\bar{\lambda}_{k-1, j} .
$$

Multiplying both sides of (41) from left with $\overline{\mathbf{v}}_{k-1, i}^{T}$ and using the orthonormality of the set $\left\{\overline{\mathbf{v}}_{k-1, j}\right\}$ gives

$$
\overline{\mathbf{v}}_{k-1, i}^{T} \mathbf{x}_{k} \mathbf{x}_{k}^{T} \overline{\mathbf{v}}_{k-1, j}=\left(\bar{\lambda}_{k-1, j}-\bar{\lambda}_{k-1, i}\right) \overline{\mathbf{v}}_{k-1, i}^{T} \mathbf{u}_{k, j}
$$

We also add a third equality using the orthonormality of the eigenvectors,

$$
\overline{\mathbf{v}}_{k-1, i}^{T} \overline{\mathbf{v}}_{k-1, j}=\delta_{i j}
$$

where $\delta_{i j}$ is the Kronecker delta. Using the perturbation of the eigenvectors and equating the terms with the order $\epsilon$, we have

$$
\mathbf{u}_{k, i}^{T} \overline{\mathbf{v}}_{k-1, j}+\overline{\mathbf{v}}_{k-1, i}^{T} \mathbf{u}_{k, j}=0 .
$$

Let $b_{i j}$ be defined as the projection of the vector $\mathbf{u}_{k, j}$ onto $\overline{\mathbf{v}}_{k-1, i}^{T}$. Then, using (48) and (46), we have

$$
b_{i j}=-b_{j i}^{T}=\frac{y_{i, k} y_{j, k}^{T}}{\bar{\lambda}_{k-1, j}-\bar{\lambda}_{k-1, i}} .
$$

Such as in [27], we assume $b_{i i}=0$, and to stabilize the estimator for very close eigenvalues, we use the following approximation

$$
b_{i j}=-b_{j i}^{T}=\frac{y_{i, k} y_{j, k}^{T}}{\max \left\{0.01 \bar{\lambda}_{1, j}, \bar{\lambda}_{k-1, j}-\bar{\lambda}_{k-1, i}\right\}} .
$$

With these assumptions, the eigenvalue decomposition of the sample correlation matrix can be summarized as

$$
\begin{aligned}
\bar{\lambda}_{k, j} & =(1-\epsilon) \bar{\lambda}_{k-1, j}+\epsilon \overline{\mathbf{v}}_{k-1, j}^{T} \mathbf{x}_{k} \mathbf{x}_{k}^{T} \overline{\mathbf{v}}_{k-1, j} \\
\overline{\mathbf{v}}_{k, j} & =\overline{\mathbf{v}}_{k-1, j}+\epsilon \sum_{i=1}^{N} b_{i j} \overline{\mathbf{v}}_{k-1, j} \\
b_{i j} & =\frac{\overline{\mathbf{v}}_{k-1, j}^{T} \mathbf{x}_{k} \mathbf{x}_{k}^{T} \overline{\mathbf{v}}_{k-1, j}}{\max \left\{0.01 \bar{\lambda}_{1, j}, \bar{\lambda}_{k-1, j}-\bar{\lambda}_{k-1, i}\right\}} .
\end{aligned}
$$

The PDL algorithm proceeds by solving (51)-(53) and using the results in (44). It has been shown that the first order perturbation can significantly reduce the computational cost of the eigenvalue decomposition of the sample correlation matrix [27]. More specifically, for fixed $j$, our algorithm needs $2 N$ multiplications to compute $\bar{\lambda}_{k, j}$ in (51),N multiplications to

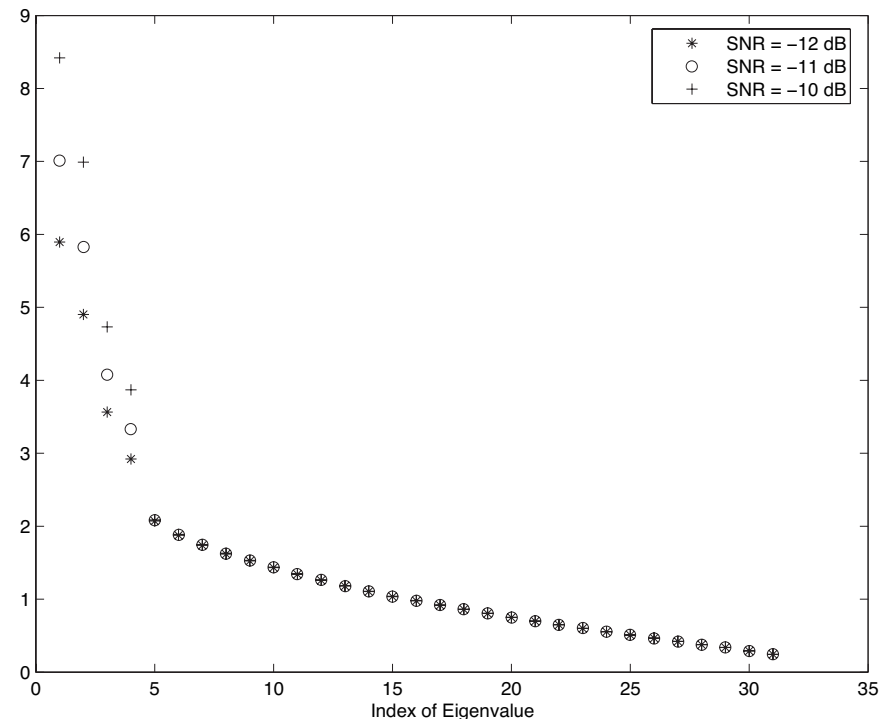

Fig. 2. The eigenvalues of the sample correlation matrix averaged over 100 independent runs. The results correspond to 3 different SNRs.

obtain $\overline{\mathbf{v}}_{k, j}$ in (52), and $N$ multiplications to calculate $\left\{b_{i j}\right\}_{i=1}^{N}$ in (53). Taking into account that $j \in\{1,2, \ldots, N\}$, the total computational complexity is $\mathcal{O}\left(N^{2}\right)$ while the computational complexity of eigenvalue decomposition and matrix inversion is indeed $\mathcal{O}\left(N^{3}\right)$.

\section{Simulation Results}

Consider a DS-CDMA system with 4 active signals using the 31-bit Gold codes. The received signal is the superposition of the signals of all users. Noise is assumed to be a white Gaussian process. The signal-to-noise ratio (SNR) is defined as the ratio of the power of any one of the signals to the power of noise. Two of the four signals are assumed to have equal SNRs ranging from $-15 \mathrm{~dB}$ to $-10 \mathrm{~dB}$. The other two signals have also equal SNRs, but their SNRs are assumed to be 3 $\mathrm{dB}$ higher than that of the first two users.

We performed 100 independent runs and found the eigenvalues of the sample correlation matrix in each run. Fig. 2 illustrates the eigenvalues of the sample correlation matrix averaged over 100 runs for three different values of the weakest source SNR. Note that the eigenvalues can be decomposed into signal and noise eigenvalues. The large eigenvalues (in this example the first 4 eigenvalues) are the signal eigenvalues. In this example, the distance between the 4th and the 5th eigenvalues is larger than the distance between the other subsequent eigenvalues. Therefore, a simple detector (denoted as EIG) selects the number of signals by comparing the difference between the consecutive eigenvalues. We will compare our proposed detector to EIG in the sequel.

The MDL and PDL techniques were used to estimate the number of signals. We have performed 100 independent runs and presented the results in Table I. In this table, three detectors have been compared. Each row in the table shows the number of times that the detector selected the corresponding model for the given SNR. Note that PDL outperforms the MDL and EIG methods. As the SNR decreases, the signal and noise eigenvalues approach each other and cannot be easily 
TABLE I

THE NUMBER OF DS-CDMA SIGNALS DETECTED BY THE EIG, MDL, AND PDL ALGORITHMS FOR 100 INDEPENDENT RUNS.

\begin{tabular}{|r|c|rrrrrr|}
\hline & & \multicolumn{7}{|c|}{ SNR $(\mathrm{dB})$} \\
\cline { 3 - 8 } & $\mathrm{m}$ & -15 & -14 & -13 & -12 & -11 & -10 \\
\hline \multirow{5}{*}{ EIG } & 1 & 43 & 36 & 32 & 26 & 22 & 18 \\
& 2 & 37 & 50 & 58 & 65 & 68 & 70 \\
& 3 & 18 & 12 & 5 & 3 & 2 & 0 \\
& 4 & 2 & 2 & 5 & 6 & 8 & 12 \\
& 5 & 0 & 0 & 0 & 0 & 0 & 0 \\
\hline \multirow{5}{*}{ MDL } & 1 & 100 & 98 & 47 & 1 & 0 & 0 \\
& 2 & 0 & 2 & 52 & 81 & 33 & 1 \\
& 3 & 0 & 0 & 1 & 17 & 47 & 14 \\
& 4 & 0 & 0 & 0 & 1 & 20 & 85 \\
& 5 & 0 & 0 & 0 & 0 & 0 & 0 \\
\hline \multirow{5}{*}{ PDL } & 1 & 45 & 9 & 0 & 0 & 0 & 0 \\
& 2 & 41 & 56 & 35 & 6 & 0 & 0 \\
& 3 & 14 & 32 & 45 & 32 & 9 & 0 \\
& 4 & 0 & 3 & 20 & 62 & 91 & 100 \\
& 5 & 0 & 0 & 0 & 0 & 0 & 0 \\
\hline
\end{tabular}

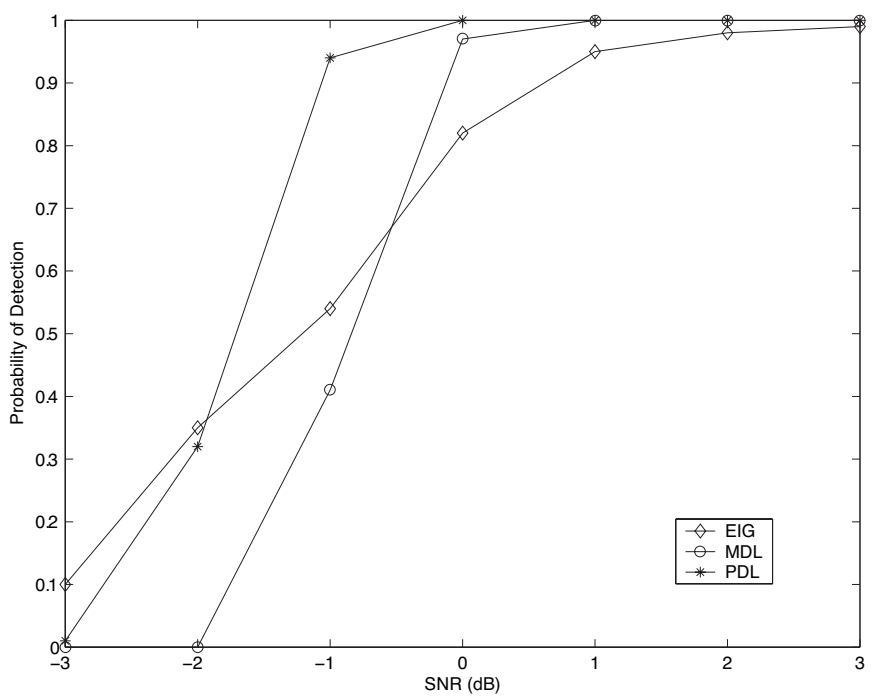

Fig. 3. The probability of resolution versus SNR for the MDL and PDL algorithms for 100 independent runs.

separated. In such cases, none of the methods can properly estimate the true number of DS-CDMA signals.

The probability of resolution for this example has been illustrated in Fig. 3. In this example, we have divided the number of times that each algorithm properly estimated the true number of sources to the total number of runs. As noticed, PDL has $1 \mathrm{~dB}$ gain over MDL and almost $2 \mathrm{~dB}$ gain over EIG.

The recursive structure of the PDL algorithm can be very useful in non-stationary environments. We study two cases where the number of sources changes inside the window of observation. We consider a window size of 300 samples with $\mathrm{SNR}=3 \mathrm{~dB}$ for all users. In the first example, we assume that the number of signals is 3 at the beginning of the window and changes to 4 at $k=100$. We compute the PDL cost for 20 independent runs and find the average of these runs. The PDL cost is computed for all $1 \leq k \leq 300$. At each time instant, we estimate the number of signals by locating the minimum PDL cost. The number of detected signals has been shown as a function of time in Fig. 4. Note that the number of detected signals changes from 3 to 4 in the vicinity of the 100th time instant.

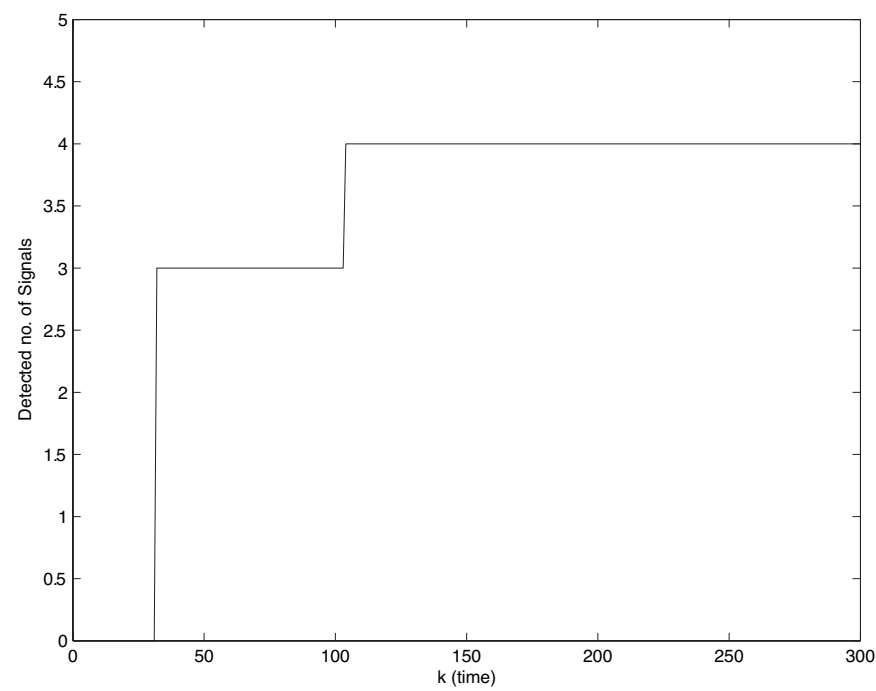

Fig. 4. The estimated number of sources as a function of the observation window. The true number of signals changes from 3 to 4 at $k=100$.

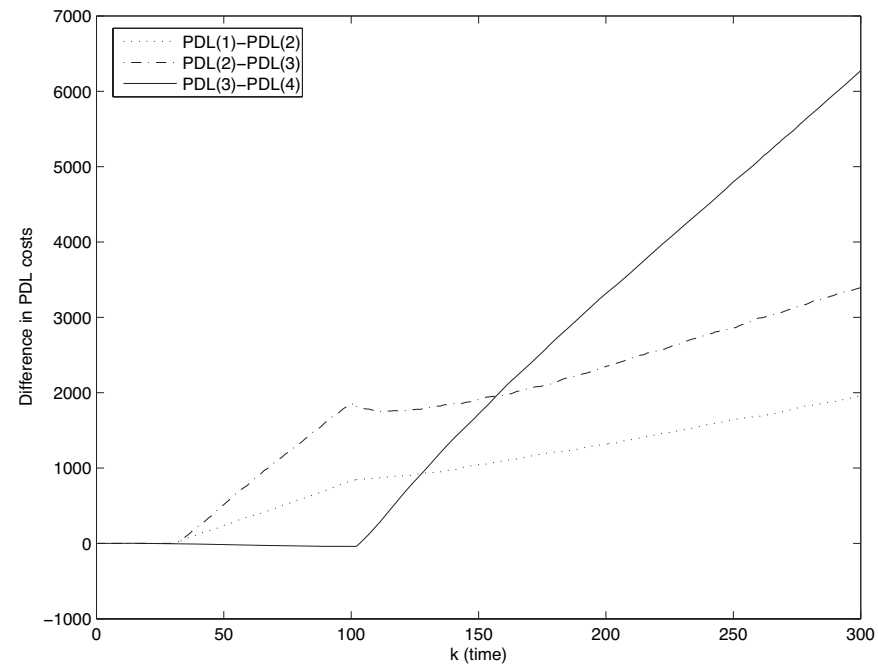

Fig. 5. The difference between the PDL terms of a model of order $m$ and the corresponding terms of the model of order $m+1$. The true number of signals changes from 3 to 4 at $k=100$.

For this example, we have also computed $\operatorname{PDL}_{m}(k)-$ $\mathrm{PDL}_{m+1}(k)$, the difference between the PDL costs of consecutive models for all time instants inside the window of observation. The results have been illustrated in Fig. 5 for three different values of $m=1,2,3$. Note an abrupt change in the slope of $\mathrm{PDL}_{3}(k)-\mathrm{PDL}_{4}(k)$ in the vicinity of $k=100$. The change indicates that the underlying model which was used for $k=1, \ldots, 100$ is not valid for the rest of the window. This figure can be used to locate the change point inside the window of observation. Since MDL operates on a batch of data, it cannot locate the change.

We also study the case where the number of signals changes from 4 to 3 at the 100th time instant. Such as before, the PDL cost is averaged over 20 independent runs. The results have been shown in Fig. 6 and Fig. 7. Note that the number of signals has not been detected properly over the window $k=101, \ldots, 300$. The reason for this malfunctioning is that the number of prominent eigenvalues of the sample correlation 


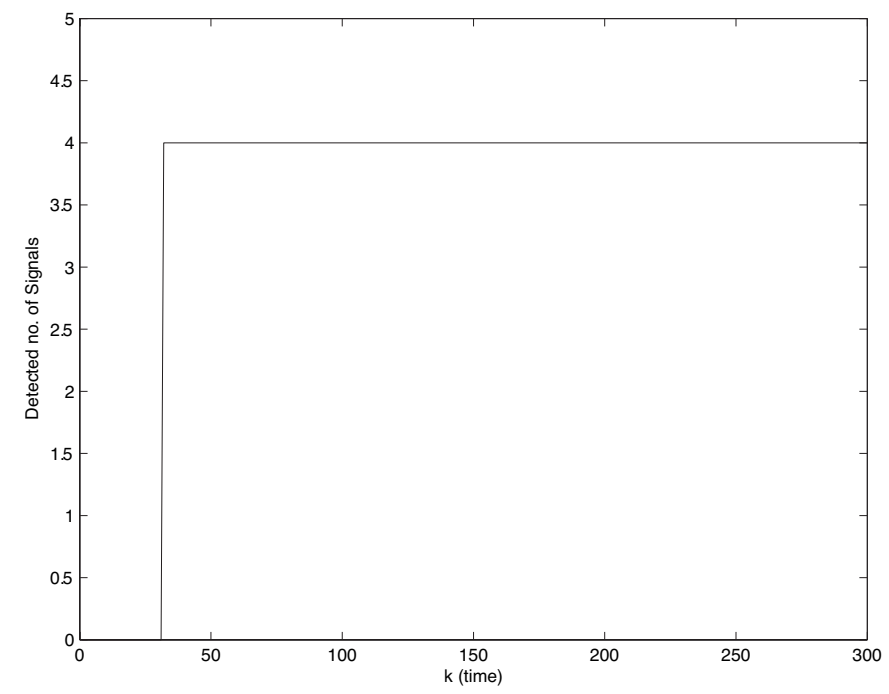

Fig. 6. The estimated number of sources as a function of the observation window. The true number of signals changes from 4 to 3 at $k=100$.

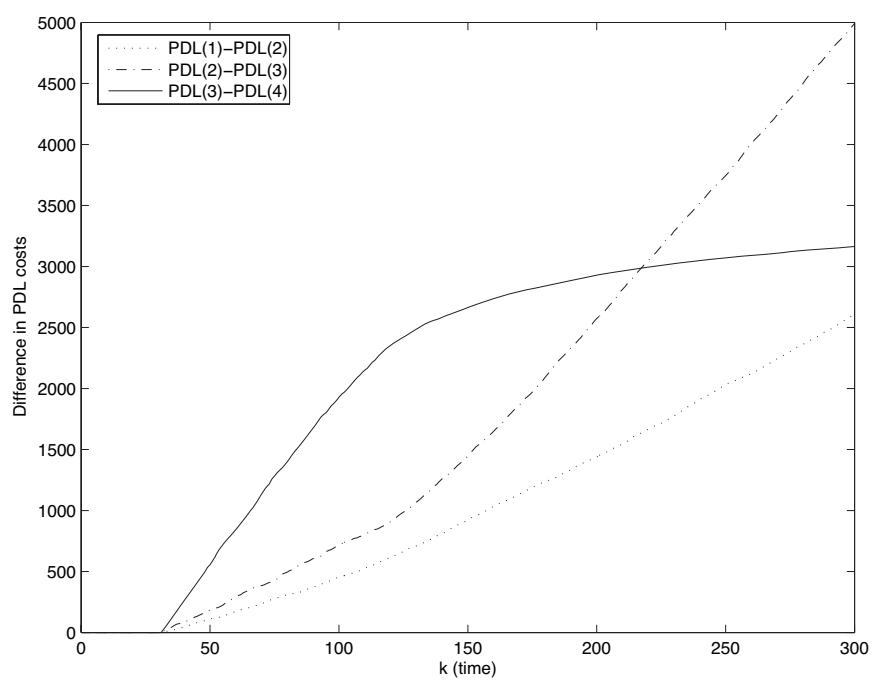

Fig. 7. The difference between the PDL terms of a model of order $m$ and the corresponding terms of the model of order $m+1$. The true number of signals changes from 4 to 3 at $k=100$.

matrix is 4 even after the actual number of signals is reduced to 3. Fig. 7 shows the difference between the PDL costs of consecutive model orders. Notice the abrupt change in the slope of the curve $\mathrm{PDL}_{3}(k)-\mathrm{PDL}_{4}(k)$. The change indicates that the underlying model is not valid and the sample correlation matrix should be reset and recomputed using the recent data.

\section{SUMMARY}

In this paper, we have introduced a new information theoretic method to estimate the number of signals in DS-CDMA networks. Our approach is based on the predictive description length (PDL). PDL is the length of a predictive code that encodes the observed data. We use the code length as a metric that describes the observation vector. The best model is the one that gives the smallest code length. The PDL cost is computed for all candidate models and the one with the smallest cost is selected as the best-fit model.
The proposed method is based on the ML estimate of the correlation matrix. To apply our technique, we do not need the signature waveform of DS-CDMA signals and only use the multiplicity of the smallest eigenvalue of the correlation matrix. Therefore, this technique can be used in blind multiuser detection where the signature waveform of signals is not known. The simulation results show that the PDL algorithm has a lower detection threshold SNR than the MDL and EIG methods.

\section{REFERENCES}

[1] S. Verdu, Multiuser Detection. Cambridge, U.K.: Cambridge Univ. Press, 1998.

[2] X. Wang and H. Poor, "Blind multiuser detection: a subspace approach," IEEE Trans. Inform. Theory, vol. 44, pp. 677-690, 1998.

[3] M. Wax and I. Ziskind, "Detection of the number of coherent signals by the MDL principle," IEEE Trans. Acoust., Speech, Signal Processing, vol. 37, pp. 1190-1196, Aug. 1989.

[4] Q. T. Zhang, K. M. Wong, P. C. Yip, and J. P. Reilly, "Statistical analysis of the performance of information theoretic criteria in the detection of the number of signals in array processing," IEEE Trans. Acoust., Speech, Signal Processing, vol. 37, pp. 1557-1567, Oct. 1989.

[5] Q. Wu and D. R. Fuhrmann, "A parametric method for determining the number of signals in narrow-band direction finding," IEEE Trans. Acoust., Speech, Signal Processing, vol. 39, pp. 1848-1857, Aug. 1991.

[6] T. E. Shrimpton and S. Schell, "Source enumeration using a signal selective information theoretic criterion," in Proc. Military Communications Conference (MILCOM), vol. 3, pp. 1092-1097, 1997.

[7] K. C. Tan, M. Zhang, and M. Wax, "Signal enumeration and direction of arrival estimation for coherent signals in correlated noise with unknown parameters," in Proc. Int. Conf. on Signal Processing (ICSP), vol. 1, pp. 339-342, 1998.

[8] A. Barron, J. Rissanen, and B. Yu, "The minimum description length principle in coding and modeling," IEEE Trans. Inform. Theory, vol. 44, pp. 2743-2760, Oct. 1998.

[9] M. H. Hansen and B. Yu, "Model selection and the principle of minimum description length," J. American Stat. Assoc., vol. 96, pp. 746-774, June 2001.

[10] P. J. Green and D. P. Taylor, "Dynamic signal enumeration algorithm for smart antennas," IEEE Trans. Signal Processing, vol. 50, pp. 1307-1314, June 2002.

[11] S. Valaee and P. Kabal, "An information theoretic approach to source enumeration in array signal processing," IEEE Trans. Signal Processing, vol. 52, pp. 1171-1178, May 2004.

[12] J. Rissanen, "Modeling by shortest data description," Automatica, vol. 14, pp. 465-471, 1979.

[13] J. Rissanen, "Stochastic complexity and modeling," Annals of Statistics, vol. 14, pp. 1080-1100, 1986.

[14] M. Wax and T. Kailath, "Detection of signals by information theoretic criteria," IEEE Trans. Acoust., Speech, Signal Processing, vol. 33, pp. 387-392, Apr. 1985.

[15] E. Fishler, M. Grosman, and H. Messer, "Determining the number of discrete alphabet sources from sensor data," EURASIP J. Applied Signal Processing, vol. 2005, pp. 4-12, Jan. 2005.

[16] J. Rissanen, Stochastic Complexity in Statistical Inquiry. World Scientific Publisher, 1989.

[17] D. H. Johnson and D. E. Dudgeon, Array Signal Processing: Concepts and Techniques. Prentice-Hall, 1993.

[18] S. Valaee, B. Champagne, and P. Kabal, "Sinusoidal signal detection using the minimum description length and the predictive stochastic complexity," in Proc. Digital Signal Processing Conference, pp. 10231026, 1997.

[19] H. Liu and G. Xu, "A subspace method for signature waveform estimation in synchronous CDMA systems," IEEE Trans. Commun., vol. 44, pp. 1364-1354, Oct. 1996.

[20] K. Zarfi, S. Shahbazpanahi, A. B. Gershman, and Z.-Q. Luo, "Robust blind multiuser detection based on the worst-case performance optimization of the mmse receiver," IEEE Trans. Signal Processing, vol. 53, pp. 295-305, Jan. 2005.

[21] X. Wang and H. V. Poor, "Blind adaptive multiuser detection in multipath CDMA channels based on subspace tracking," IEEE Trans. Signal Processing, vol. 46, pp. 3030-3044, Nov. 2005. 
[22] S. Shahbazpanahi and A. B. Gershman, "Robust blind multiuser detection for synchronous CDMA systems using worst-case performance optimization," IEEE Trans. Wireless Commun., vol. 3, pp. 2232-2245, Nov. 2004.

[23] R. Schmidt, "A signal subspace approach to multiple emitter location and spectral estimation," Ph.D. thesis, Stanford University, 1981.

[24] J. Rissanen, "Fisher information and stochastic complexity," IEEE Trans. Inform. Theory, vol. 42, pp. 40-47, Jan. 1996.

[25] T. W. Anderson, "Asymptotic theory for principal component analysis," Ann. Math. Stat., vol. 34, pp. 122-148, 1963.

[26] S. Valaee, "An information theoretic transmitter enumerator for DSCDMA wireless networks," in Proc. IEEE Globecom 2004, Dec 2004.

[27] B. Champagne, "Adaptive eigendecomposition of data covariance matrices based on first-order perturbations," IEEE Trans. Signal Processing, vol. 42, pp. 2758-2770, Oct. 1994.

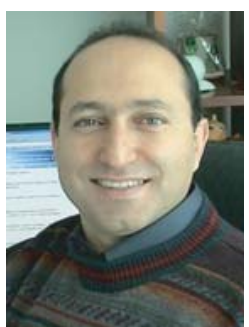

Shahrokh Valaee (S88,M00,SM02) received the Ph.D. degree from McGill University, Montreal, in Electrical Engineering. From 1994 to 1995, he was a Research Associate at INRS Telecom in Montreal. From 1996 to 2001, he was an Assistant Professor of Electrical Engineering, at Tarbiat Modares University in Teheran. Since September 2001, he has been an Associate Professor of Electrical and Computer Engineering at the University of Toronto and holds the Nortel Institute Junior Chair of Communication

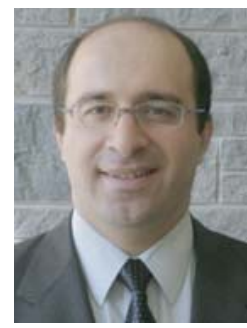

Shahram Shahbazpanahi (M02) was born inSanandaj, Kurdistan, Iran. He received the B.Sc., M.Sc., and Ph.D. degrees in electrical engineering from Sharif University of Technology, Tehran, Iran, in 1992, 1994, and 2001, respectively. From September 1994 to September 1996, he was a faculty member with the Department of Electrical Engineering, Razi University, Kermanshah, Iran. From July 2001 to March 2003, he was a Postdoctoral Fellow with the Department of Electrical and Computer Engineering, McMaster University, Hamilton, Ontario, Canada. From April 2003 to September 2004, he was a Visiting Researcher with the Department of Communication Systems, University of Duisburg-Essen, Duisburg, Germany. From September 2004 to April 2005, he was a Lecturer and Adjunct Professor with the Department of Electrical and Computer Engineering, McMaster University. Since July 2005, he has been with the Faculty of Engineering and Applied Science, University of Ontario Institute of Technology, Oshawa, Canada, where he holds an Assistant Professor position. His research interests include statistical and array signal processing, space-time adaptive processing, detection and estimation, smart antennas, spread spectrum techniques, MIMO communications, DSP programming, and hardware/real-time software design for telecommunication systems. Dr. Shahbazpanahi is currently serving as Associate Editor for the IEEE Transaction on Signal Processing and IEEE Signal Processing Letters. $\mathrm{He}$ is also a member of the Sensor Array and Multichannel (SAM) Technical Committee of the IEEE Signal Processing Society. 